\title{
Right ventricular outflow tract PVC during COVID-19 infection. Is there a fortuitous link?
}

\author{
Amato Santoro ${ }^{1}$ \\ ${ }^{1}$ Azienda Ospedaliera Universitaria Senese
}

April 28, 2020

\begin{abstract}
Covid-19 is a respiratory illness caused by coronavirus SARS-1CoV-2. High levels of cytokines, particularly interleukin 6 (IL-6) are found in Covid-19 patients. IL-6 induce sympathetic hyperactivation that trig premature ventricular contraction (PVC). Ten point five percentage of Covid-19 patients of our Hospital developed right outflow tract (RVOT) PVC associated to high value of IL-6. After administration of Tocilizumab (IL-6 antagonist) once the infection was resolved, the PVC disappeared. Are the increased values of IL-6 an aspect of myocardial RVOT initial inflammation and fibrosis, or the effect of the high pulmonary pressure and RV pressure overload that determine myocardial stretch and subsequent PVC? The question of whether the elevation of this inflammatory biomarker is the cause or consequence of ventricular arrhythmia manifestation.
\end{abstract}

\section{Hosted file}

RVOT COVID. doc available at https://authorea.com/users/313699/articles/444160-right-ventricularoutflow-tract-pvc-during-covid-19-infection-is-there-a-fortuitous-link

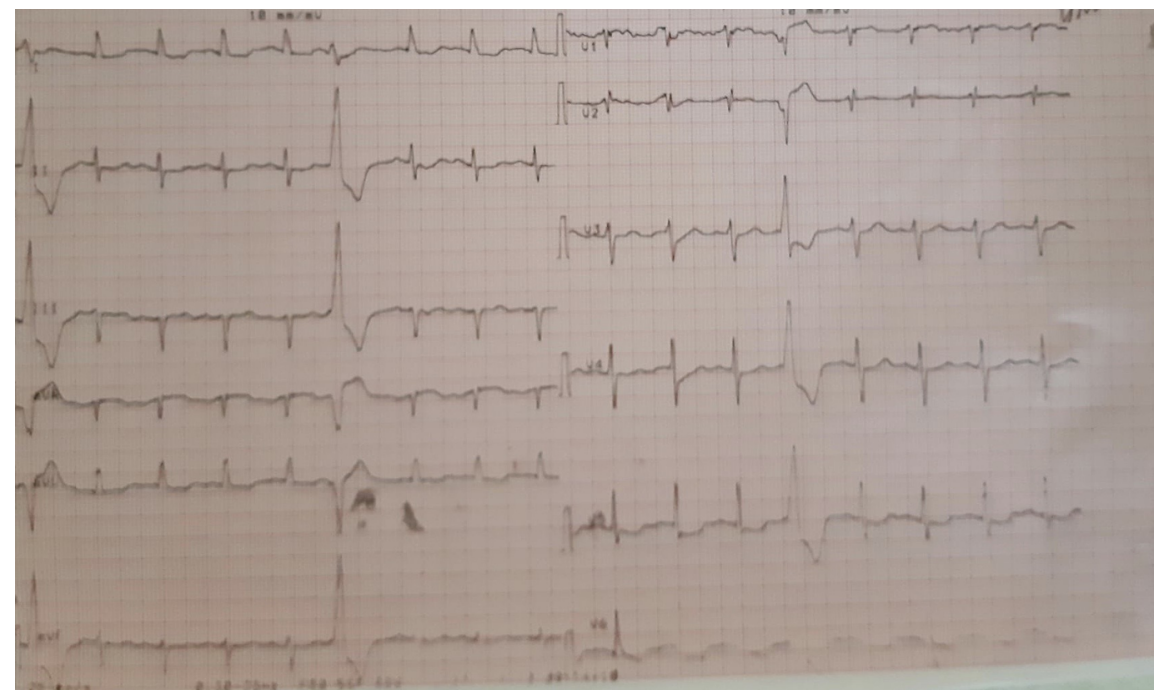




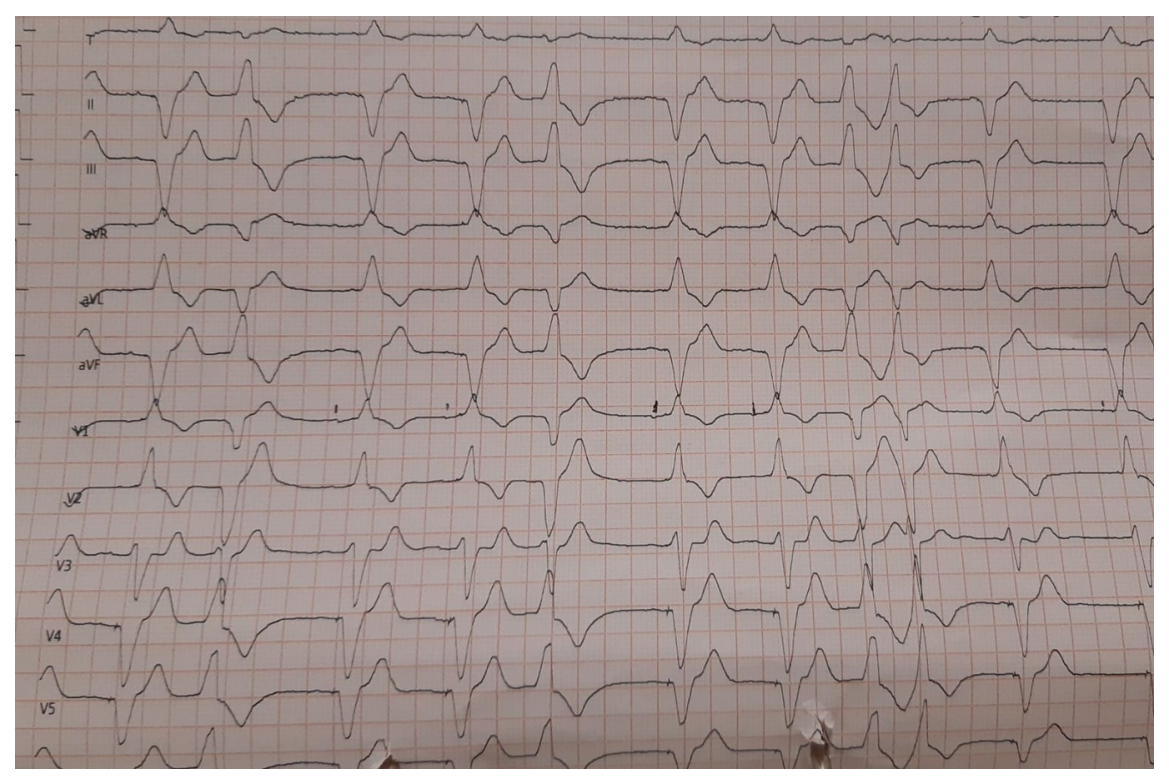

